\title{
Study on the Influence of Absorbing Interference Cloud on Radar Effectiveness Based on HFSS
}

\author{
Yizhi GE, Xingwei $\mathrm{LI}^{1}$, Xianfa ZHAO and Jiazhe ZHANG \\ College of Intelligence Science and Technology, National University of Defense \\ Technology, China
}

\begin{abstract}
In order to make up for the deficiency of traditional passive jamming, a new electronic jamming mode is proposed, which mainly absorbs electromagnetic waves. Firstly, based on the traditional interference corridor model of radar electromagnetic signal power attenuation, the absorber as a whole is analyzed and its interference mechanism is obtained; With reference to the idea of studying the electromagnetic characteristics of stealth metamaterials by Floquet port method, the electromagnetic simulation software HFSS is used to establish an electromagnetic model for simulation verification, and the scaled method is used to simplify the model. The experimental results are consistent with the theory, which shows that the interference mechanism and the interference simulation model are correct.
\end{abstract}

Keywords. HFSS simulation, interference cloud, scaling method, metamaterial absorber

\section{Introduction}

In the field of electronic countermeasures, passive jamming is not only the earliest application, but also the most widely used jamming mode because of its advantages of simple equipment and convenient implementation. However, with the development of electronic countermeasures technology, the limitations of passive jamming mainly relying on traditional chaff, such as passivity of jamming, easy recognition by new radar system, and requirement for jamming space position, have emerged [1]. Therefore, it is of practical significance to study new passive jamming modes. Earlier, Zheng Weiping and others studied the interference of carbon fiber to millimeter wave radar in literature [2], and Han Chao and others put forward absorbing chaff based on nano materials and carried out experimental test[3]. In recent years, expanded graphite [4,5], foam cloud $[6,7,8]$, modified fiber $[9,10]$ and other interference absorbing materials have also been developed, improved and applied in passive interference field. At present, the research mainly focuses on the reduction of RCS of targets by absorbing materials, while the influence of interference clouds formed by interfering materials on the echo characteristics of radar electromagnetic waves is rarely involved. Therefore, on the basis of the power attenuation model of radar electromagnetic signal caused by interference

${ }^{1}$ Corresponding Author, Xingwei LI, College of Intelligence Science and Technology, National University of Defense Technology, China; E-mail: lxw_mmoml@nudt.edu.cn. 
corridor formed by traditional chaff in reference [11], this paper uses finite element software to establish a wave-absorbing interference cloud model to evaluate the wave-absorbing efficiency of radar electromagnetic wave.

\section{Model Derivation}

Electronic jamming of absorbing waveform is similar to conventional passive jamming, which is mainly used for attack and retreat of shield carrier. The schematic diagram is shown in figure 1 below.

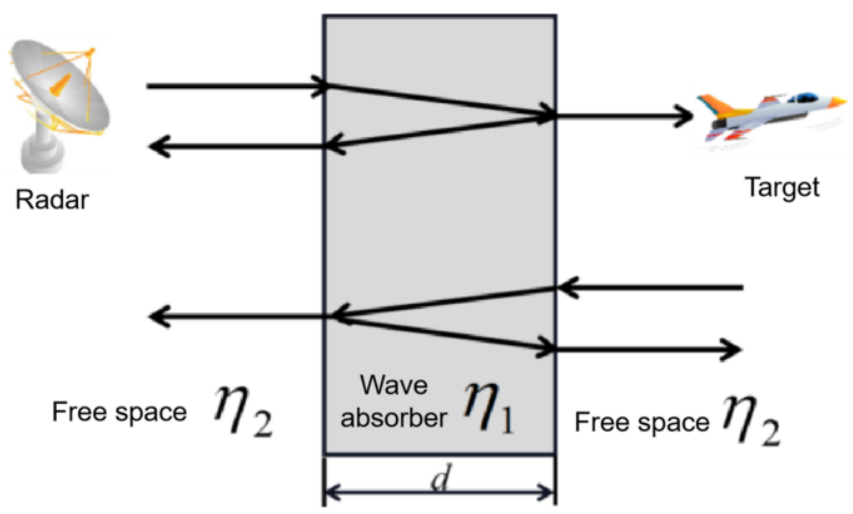

Figure 1. Schematic diagram of influence of absorbing interference cloud on radar electromagnetic wave

Microscopically, the interference cloud is composed of various wave-absorbing particles, which are distributed dynamically in space, non-uniform and constantly changing dynamically. However, in order to simplify the problem, Gaussian is adopted to deal with the flux problem, and the interference cloud as a whole is regarded as a uniformly distributed isotropic material layer. When radar electromagnetic wave is incident on interference cloud, it will produce reflection and transmission. According to the principle of energy conservation, the energy of incident wave is equal to the sum of the energy of reflected wave and transmitted wave and the lost energy, namely

$$
E=E_{T}+E_{R}+E_{C}
$$

Where $E$ is the energy of incident electromagnetic wave, $E_{T}$ is the energy of transmitted wave, $E_{R}$ is the energy of reflected wave and $E_{C}$ is the energy of loss. According to the theory of electromagnetic wave transmission line [12]: 


$$
\begin{aligned}
E_{T}= & E \cdot t_{01} t_{12} e^{-\gamma d}+E \cdot t_{01} t_{12} r_{01} r_{12} e^{-2 \gamma \cdot d} \\
& +E \cdot t_{01} t_{12}\left(r_{01} r_{12} e^{-2 \gamma \cdot d}\right)^{2} \\
& +E \cdot t_{01} t_{12}\left(r_{01} r_{12} e^{-2 \gamma \cdot d}\right)^{3}+\ldots \\
= & \frac{E \cdot t_{01} t_{12} e^{-\gamma \cdot d}}{1-r_{01} r_{12} e^{-2 \gamma \cdot d}}
\end{aligned}
$$

Where $\mathrm{d}$ is the thickness of absorber, $t_{i j}$ and $r_{i j}$ represent the reflection coefficient and transmission coefficient from $\mathrm{i}$ space to $\mathrm{j}$ space, $\gamma$ is the complex transmission coefficient of absorber, $\gamma$ the real part indicates the ability of absorber to lose electromagnetic waves, and the imaginary part indicates the phase shift. In the same way, you can get:

$$
\begin{aligned}
E_{R}= & E \cdot r_{01}+E \cdot t_{01} t_{10} r_{12} e^{-2 \gamma \cdot d} \\
& +E \cdot t_{01} t_{10}\left(r_{01} r_{12} e^{-2 \gamma \cdot d}\right)^{2} \\
& +E \cdot t_{01} t_{10}\left(r_{01} r_{12} e^{-2 \gamma \cdot d}\right)^{3}+\ldots \\
= & E \cdot r_{01}+\frac{E \cdot t_{01} t_{10} e^{-2 \gamma \cdot d}}{1-r_{01} r_{12} e^{-2 \gamma \cdot d}}
\end{aligned}
$$

The following formulas can be used for reflection coefficient and transmission coefficient(4) It can be calculated

$$
\left\{\begin{array}{l}
r_{i j}=\left|\frac{\eta_{i}-\eta_{j}}{\eta_{i}+\eta_{j}}\right| \\
t_{i j}=\left|\frac{2 \eta_{i}}{\eta_{i}+\eta_{j}}\right|
\end{array}\right.
$$

Where $\eta_{i}$ and $\eta_{i}$ represent the characteristic impedance of the material. And the complex transmission coefficient $\gamma$ of the absorber is

$$
\gamma=j \omega \sqrt{\mu_{C} \varepsilon_{C}}
$$

The $\varepsilon_{C}, \mu_{C}$ are the equivalent dielectric constant and the equivalent permeability of the material, which directly affect the complex transmission coefficient of the absorber $\gamma$. The real part of is $\gamma$ called attenuation constant, and the imaginary part is phase shift constant. 
According to formulas (1) (5), the variation of power transmission loss and power reflection loss with the thickness of absorber can be obtained as shown in figure 2 below. The abscissa is the thickness, and the ordinate is the ratio of field strength.

As can be seen from figure 2: With the increase of the thickness of the absorber, the transmission loss decreases from 1 to close to 0 ; However, the reflection loss tends to decrease in oscillation, and finally tends to be stable. It shows that with the increase of thickness, the absorbing effect of the absorber on electromagnetic wave is strengthened, while the scattering effect is weakened to a certain fixed value, while the rest electromagnetic wave energy is absorbed completely.

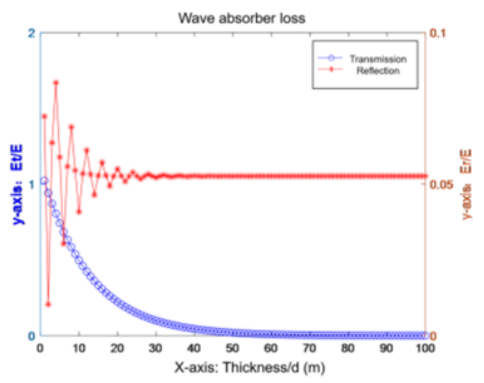

Figure 2. Reflection loss and transmission loss

\section{Simulation.}

\subsection{Electromagnetic Software Application Base on Fem}

Now, it is necessary to verify the above derivation by experiments. When dealing with complex electromagnetic field problems, the commonly used method is to use simulation software to model and solve them. Among many solving methods, Finite Element Method (FEM) is an approximate numerical method developed with the rapid development of modern computers. It can divide the solution domain into many interconnected subdomains called finite elements, so that complex problems can be solved after being replaced by many simpler problems. FEM not only has high calculation accuracy and is easy to understand and master, but also can describe various complex shapes, and has become the mainstream engineering analysis method in the industry. For example, the High Frequency Structure Simulator (HFSS), which uses FEM to deal with microwave engineering problems, is a typical representative of the application of finite element method in electromagnetic field. As the world's first commercial three-dimensional electromagnetic field simulation software, HFSS has high reliability and easy operation, and can efficiently design various high-frequency structures, including RF and microwave components, antennas and antenna arrays, and has become the first choice tool for analysis and design in the electromagnetic field.

The specific steps of designing electromagnetic model with software HFSS are as follows: Physical and geometric modeling using electromagnetic simulation software, including various models including radar and absorbing interference cloud; The material properties and excitation conditions of the absorber are set. Then, the geometric model of the target is meshed, and the reflectivity and transmittance of the target are 
approximately solved by the corresponding electromagnetic calculation method. The specific simulation design flow is shown in figure 3 [13].

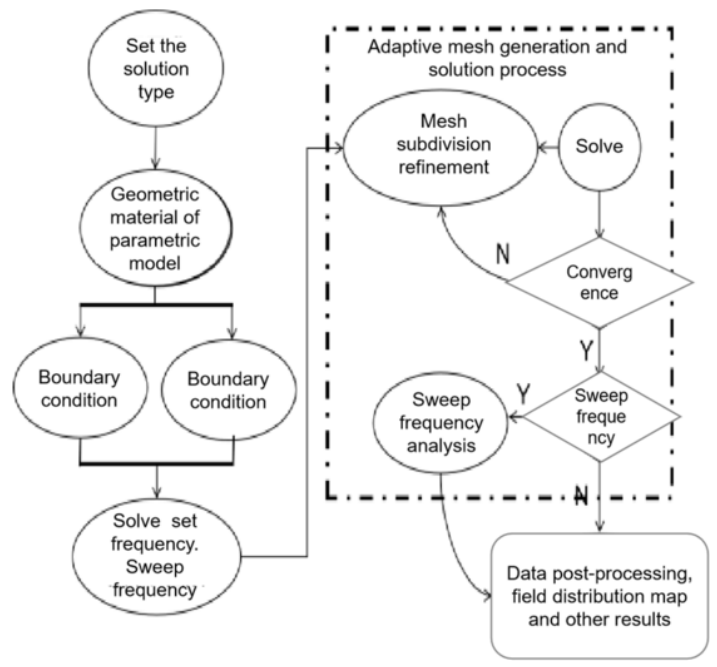

Figure 3. Flow chart of HFSS calculation

Because the traditional finite element software HFSS has low simulation efficiency and poor accuracy for electrically large-scale models, it is necessary to simplify the models appropriately by using the scaling method.

\subsection{Scale Model Simulation of Electromagnetic System}

In actual electromagnetic measurement, especially in the process of radar cross section measurement, it is often difficult to measure the electromagnetic characteristics of large targets due to problems in technology, conditions and economy. Therefore, based on the linear theory of Maxwell equation, the simulation theory of physical scaled model of electromagnetic system is put forward and widely used in electromagnetic measurement experiments.

According to the classical electromagnetic similarity law put forward by Stratton in 1941, it can be known that the radar characteristics of the simulation system are the same as those of the prototype system (i.e., the full-scale system) if the geometric size, operating wavelength, electromagnetic field strength and other parameters are scaled down accordingly [14].

Based on this similarity theory, in the process of electromagnetic simulation modeling, the model can be appropriately reduced to a reasonable size, so as to keep accurate similarity with the original system, thus reducing the errors caused by the oversize model.

In the actual simulation process, because the wavelength is reduced in equal proportion, the simulation enters the high frequency band, and the calculation amount and error are still large. At the same time, for some absorbing materials, the electromagnetic characteristic parameters, such as magnetic permeability, do not meet the linear change rule, which further affects the accuracy and calculation of simulation work. 
According to formulas (1) (5), the change rule of figure 4 can be obtained by calculation: when other conditions are unchanged, when the frequency changes by 10 times, when the thickness changes by 10 times, the reflection loss and transmission loss still keep the same trend. It can be seen that: (1)Under the same attenuation requirements, the higher the radar frequency, the easier the electromagnetic loss is, and the required absorbing cloud becomes thinner in equal proportion; (2)The attenuation trend of electromagnetic waves at different frequencies is the same without changing the material characteristics.

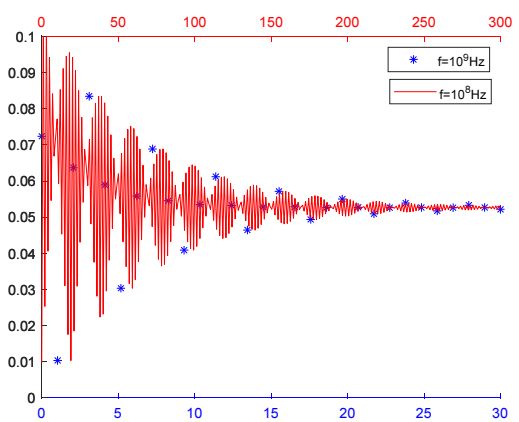

(a).Reflection loss

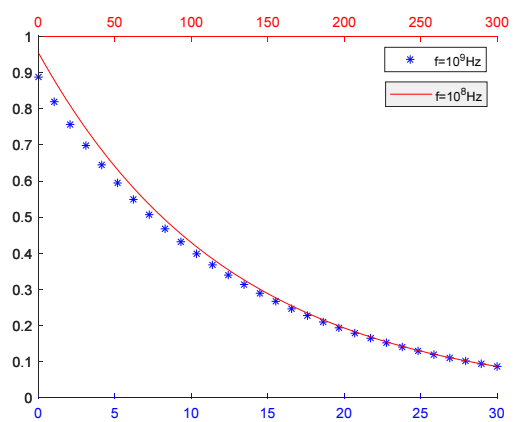

(b). Transmission loss.

Figure 4. Influence of different wavelengths on absorbing interference with other parameters unchanged

Therefore, the interference model can be further simplified, that is, the wavelength and material properties can remain unchanged, and its theoretical basis is still the linear similarity of electromagnetic theory. This simplification reduces the calculation amount and the simulation difficulty.

\subsection{Radar Wave Jamming Model}

In the study of electromagnetic waves, it is common to use HFSS to calculate the transmittance/reflectivity of absorbing materials, mainly focusing on waveguide devices, periodic structures and scatterers, such as frequency selective surfaces (FSS). Commonly used methods include waveguide transmission method, dispersion mode method and Floquet port method [15]. The accuracy of calculation results is closely related to the definition of material parameters, the selection of boundary conditions and mesh generation.

Based on FSS two-dimensional simulation method, the simulation of absorbing interference is to design a three-dimensional simple scaled model of interference screen or interference cloud according to the actual situation of interference application. Unlike Floquet port used directly in the past, the three-dimensional simulation model needs to design two groups of antenna dipoles to simulate radar, and transmit and receive electromagnetic waves respectively. For the specific principle, please refer to the transmittance/reflectivity test of absorbing materials ${ }^{[16]}$, as shown in figure 5 below. 


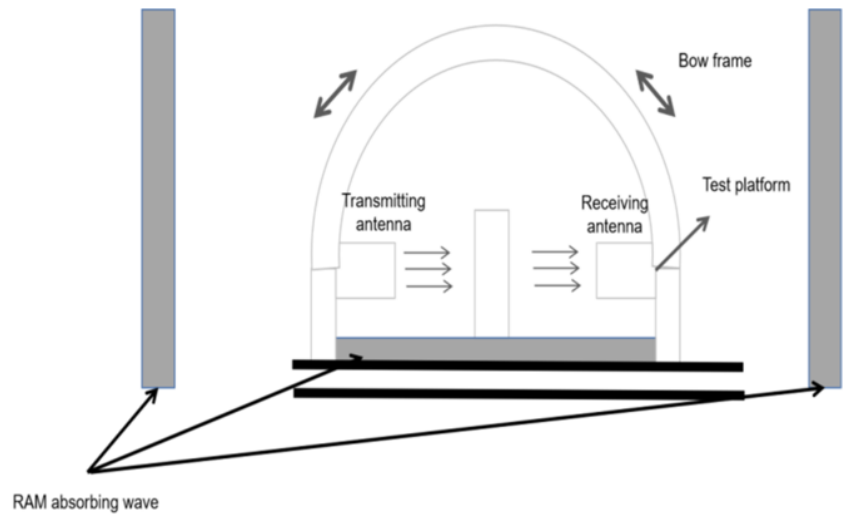

Figure 5. Schematic diagram of material transmittance/reflectivity test.

Creating interference model on HFSS19.0 platform specifically includes designing dipole antenna as radiating element, using lumped port to excite and feed, and working band is L band; On this basis, add wave-absorbing cloud and set the electromagnetic characteristics of material cloud; The designed reduction ratio is 1: 1000; The boundary of the whole model uses PML boundary to absorb redundant electromagnetic waves and simulate infinite space. The specific design is shown in figure 6 .

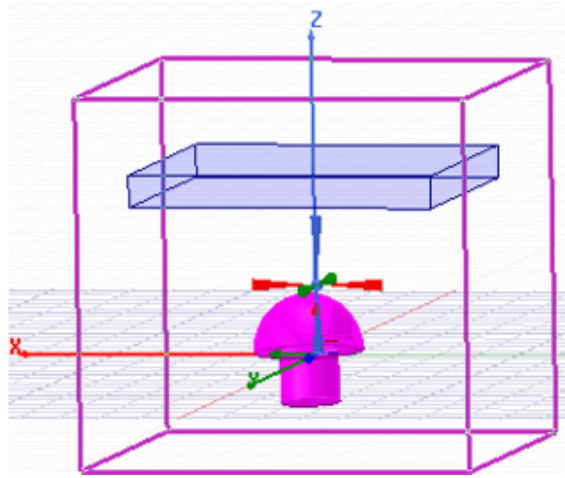

(a).Single port reflection model.

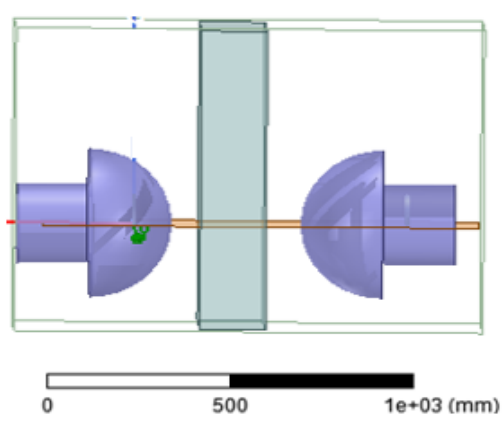

(b). Two-port transmission model.

Figure 6. HFSS absorbing interference model

According to the definition of the parameters of $\mathrm{S}$, if only one port has excitation as shown in figure 6(a), $\left[\operatorname{Mag}\left(S_{11}\right)\right]^{2}$ is the power reflectivity, while for the two-port network (see figure $6(\mathrm{~b}))\left[\operatorname{Mag}\left(S_{11}\right)\right]^{2}$ is the power transmittance (transmission rate). The experimental results can be obtained.

\section{Experimental Results and Analysis}

For the transmission model, the distance between the dipole antennas is $500 \mathrm{~mm}$ (that is, the actual $500 \mathrm{~m}$ ), the thickness of the absorbing cloud is 0 (that is, no) to $100 \mathrm{~mm}$, and the scanning step interval is $5 \mathrm{~mm}$; For the reflection model, the thickness of absorbing cloud is set to 0.1 to $20 \mathrm{~mm}$, and the step interval of scanning is set to 0.1 
$\mathrm{mm}$. The ratio of the converted echo impedance s11, reverse isolation s12/s21 and the thickness change result of the absorbing cloud to the theoretical value is shown in the following figure 7 .

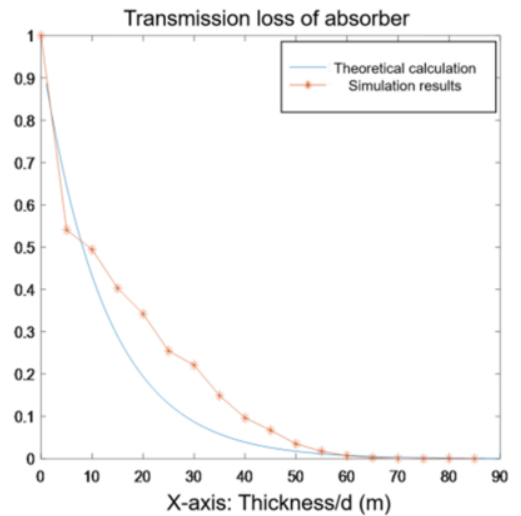

(a).Two-port transmission model.

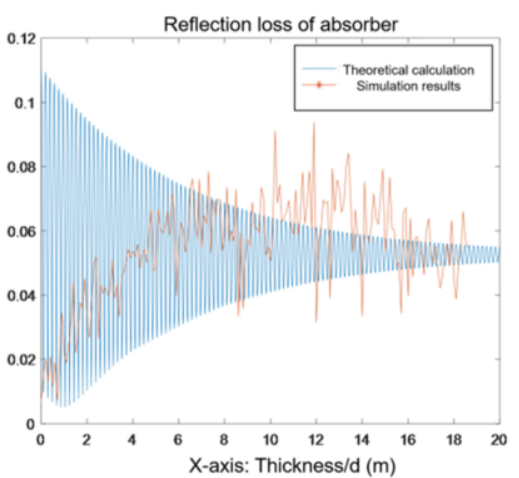

(b). Single port reflection model

Figure 7. Experimental result

It can be seen from fig. 7(a) that the simulation results of the transmission model are in good agreement with the theoretical values, that is, with the increase of the thickness, the electromagnetic wave intensity transmitted by the absorbing cloud decreases continuously.

As for the reflection model results (see fig. 7(b)), the simulation results greatly oscillate, and the reflected electromagnetic wave energy first increases and then stabilizes at a fixed value with the increase of thickness, which is roughly consistent with the trend of theoretical values. The above simulation results are in line with the actual cognition: the reflected electromagnetic wave increases to the maximum reflection value with the increase of the total amount of scatterers; Inconsistent parts with theoretical values include oscillation and theoretical values with slightly faster growth trend, and the analysis reasons may include model errors and insufficient absorption effect of PML.

\section{Conclusion}

In this paper, the absorbing jamming cloud is regarded as a single-layer absorber, and its jamming mechanism to radar electromagnetic wave is analyzed, and the influence of different thickness and frequency on jamming efficiency is explored. HFSS establishes a simple scaled model for simulation. The variation law of reflection loss with thickness in the simulation results is basically consistent with the theory, which proves the correctness and reliability of the model, and lays a foundation for evaluating the actual combat performance and application of absorbing interference. 


\section{References}

[1] Ja Fe. Preparation of Absorbing Foil by Wet Chemical Method and Study on Radar Wave Attenuation Performance [D]. Beijing: China building materials science research institute, 2015.

[2] Zheng Weiping et al. Study on interference effect of short carbon fiber silk cloud on millimeter wave [J]. research on chemical defense, 2000.(1):6 10.

[3] an Chao, Zhao Guozhi and Huang Xiaozhong. Study on millimeter wave absorbing chaff [J]. Journal of Missile and Guidance, 2006(S6):484-485.

[4] Nandi Sunny,Das Shyamal K.. A simple strategy to improve the electrochemical performance of rechargeable aqueous Al-graphite battery [J]. Materials Letters, 2021, 301.

[5] Duan Wenjing, Zhang Long, Wang Xiaorui, Wan Xiaona, Li Hui, Du Xueyan Preparation and microwave absorbing properties of expanded graphite/polyaniline composites [J]. Functional Materials, 2018, 49 (05): 5115-5119+5125.

[6] Jin Liangan, Tian Hengdou, Zhan Xichen, Shi Kan. Interference characteristics of colloidal gas foam on IR/MMW [J]. Journal of Infrared and Millimeter Wave, 2010, 29 (01):19-22.

[7] Xie Chunsi, Wang Minqing, Jin Liangan, Zhang Yuan. Theoretical and experimental study on improving the jamming effect of foam cloud on seeker [J]. Acta Microwave Sinica, 2008 (03):10-13.

[8] Jin Liangan, Wang Xiaotong, Fu Jianguo, Yang Changqing. Experimental study on interference of special foam cloud to infrared in common frequency band [J]. Laser and Infrared, 2004(04):279-281.

[9] Koch. Smoke screen aerosols opaque in visible-IR-milli-meter-band spectracontaining coated microfiber dipoles. US6578492, 2003

[10] Chen Xin, Pan Gongpei, Zhao Jun, Cao Chuanxin. Performance of nickel-coated bamboo fiber as millimeter wave interference material [J]. Journal of PLA University of Science and Technology (Natural Science Edition), 2010,11 (6): 664-667

[11] Hu Song, Lu Jingshuang, Xu Tong. Effectiveness evaluation of chaff suppression jamming on radar signal attenuation [J]. Journal of Jilin University (Information Science Edition), 2008(03):259-263.

[12] Edited by Guo Huiping and Liu Xueguan. Electromagnetic field and electromagnetic wave [M]. Xi 'an: xidian university Press, 2015

[13] Edited by Li Mingyang and Liu Min. HFSS antenna design. 2nd edition [M]. Beijing: Electronic Industry Press, 2014

[14] Jiang Wenting, Wu Dewei, Jing He. Overview of key technologies of target radar cross section scaling measurement $[\mathrm{J}]$. journal of information engineering university, 013,14(02):184-188.

[15] Xiao Hong. Basic structure analysis of anisotropic medium [D]. Harbin Engineering University, 2010.

[16] Wang Guijuan, Song Lijun, Liu Hao, Zhang Furong. Measurement of reflection loss of microwave absorbing materials in microwave darkroom [J]. Safety and Electromagnetic Compatibility, 2017(01):35-37. 